DOI: $10.35381 /$ r.k.v4i1.371

\title{
Estrategias Administrativas y Financieras: herramientas clave para una gestión eficiente
}

\section{Administrative and Financial Strategies: key tools for efficient management}

\author{
Edgar Orlando Mosquera Choco \\ emosquerac@psg.ucacue.edu.ec \\ Universidad Católica de Cuenca \\ Ecuador \\ https://orcid.org/0000-0001-5698-0253 \\ Juan Carlos Erazo Álvarez \\ jcerazo@ucacue.edu.ec \\ Universidad Católica de Cuenca \\ Ecuador \\ https://orcid.org/0000-0001-6480-2270 \\ Cecilia Ivonne Narváez Zurita \\ inarvaez@ucacue.edu.ec \\ Universidad Católica de Cuenca \\ Ecuador: \\ https://orcid.org/0000-0002-7437-9880
}

Recibido: 10 de agosto de 2019

Aprobado: 30 de agosto de 2019

\section{RESUMEN}

El presente artículo, tiene como objetivo establecer estrategias administrativas y financieras con el fin de disminuir el nivel de endeudamiento en la empresa SERSEIVI Servicio de Seguridad y Vigilancia Cía. Ltda. Se utilizaron los métodos Descriptivo-Explicativo ya que se analizó las causas que originan la situación de estudio. El tipo de investigación empleado es no experimental, el enfoque es cualitativo a través de la investigación exploratoria, recopilando datos aplicando varias técnicas como cuestionarios, observaciones, entrevistas, foro grupal, etc. con la finalidad de llevar a cabo un análisis más amplio y lograr una mejor comprensión de las situaciones. Basado en los resultados obtenidos 
se aplicó el enfoque cuantitativo evidenciado en los resultados a través de los cuales fue posible definir una propuesta que permita disminuir el sobre endeudamiento.

Descriptores: Estrategias; Administración; Finanzas; Gestión eficiente; Herramientas

\section{ABSTRACT}

The goal of this article is to establish administrative and financial strategies in order to reduce the level of indebtedness in the company SERSEIVI Security and Surveillance Service Cía. Ltda. Descriptive-Explanatory methods were used since the causes that originated the study situation were analyzed. The type of research used is non-experimental, the approach is qualitative through exploratory research, collecting data applying various techniques such as questionnaires, observations, interviews, group forum, etc. in order to carry out a broader analysis and achieve a better understanding of situations. Based on the results obtained, the quantitative approach evidenced in the results was applied, through which it was possible to define a proposal that allows reducing the indebtedness.

Descriptors: strategies; administration; finance; efficient management; tools

\section{INTRODUCCIÓN}

En los últimos años en el Ecuador ha sido evidente un auge de las empresas dedicadas a los servicios de seguridad y vigilancia como se puede apreciar en la Tabla 1, Ledesma (2018), Ministro de Trabajo, afirma que sólo hasta el primer semestre del año 2018 existían un registro de más de 804 compañías legalmente constituidas, sin embargo, esta cifra podría incrementar considerando que hay un gran número que son informales. De acuerdo a lo manifestado por el Ministerio del Interior (2018), entre todas ellas, formales e informales, han creado plazas de trabajo para un número aproximado de 120000 personas a nivel nacional.

\section{Compañías legalmente constituidas} Tabla 1

\begin{tabular}{cc}
\hline Año & \# de empresas \\
\hline 2011 & 478 \\
2012 & 506 \\
\hline
\end{tabular}




\begin{tabular}{ll}
\hline 2013 & 517 \\
2014 & 494 \\
2015 & 570 \\
2016 & 574 \\
\hline
\end{tabular}

Fuente: Ministerio del Interior (2017)

Son varias las entidades en el Ecuador que otorgan permisos, controlan el funcionamiento y ejecución de las actividades de las empresas, algunas de ellas son: la Superintendencia de Compañías (SC), Servicio de Rentas Internas (SRI), Instituto Ecuatoriano de Seguridad Social (IESS), Policía Nacional (PN), Ministerio del Interior y del Trabajo. Según datos del Ministerio del Interior con corte al mes de diciembre del año 2017 existían un total de 894 empresas de seguridad en el país, pero no todas ellas están constituidas legalmente, ver Tabla 2.

\section{Autorización Empresas de Seguridad a nivel nacional}

Tabla 2

\begin{tabular}{lc}
\hline \multicolumn{1}{c}{ Autorización } & \# de empresas \\
\hline Vigente & 556 \\
Caducadas & 203 \\
En trámite & 135 \\
\hline
\end{tabular}

Fuente: Ministerio del Interior (2017)

Entre los aspectos más relevantes que son considerados para el correcto funcionamiento están el contar con permisos para el uso de armamento letal, armamento de fogueo, equipos de protección, capacitaciones continuas al personal operativo, horas a laborar, afiliación al seguro social, etc.

En la ciudad de Cuenca existen varias empresas de seguridad y vigilancia que se encuentran operando hoy en día, las cuales ya no ofrecen únicamente servicios de seguridad física, sino que, también han ampliado su portafolio de servicios a otros, tales como: 
seguridad electrónica, personal de investigación, transporte de valores, tanto a empresas privadas como públicas y personas naturales.

Los operativos de control que se realizan en la ciudad por parte de la Policía Nacional son continuos, verificando que las empresas cuenten con los documentos habilitantes para su correcto funcionamiento, así como también, que los guardias de seguridad porten la credencial emitida por el Ministerio del Interior, que las armas letales cuenten con sus permisos de tenencia caso contrario son retiradas del sitio.

La empresa Servicios de Seguridad y Vigilancia SERSEIVI Cía. Ltda. tiene su oficina matriz en la ciudad de Cuenca, fue constituida en el año 2003 con el objetivo de ofrecer un servicio integral, especializado en protección para la industria y comercio, bajo un esquema de absoluta confiabilidad que, aunado a experiencia y capacitación, conforman un servicio óptimo de seguridad privada.

Su misión es la de prestar servicios de seguridad física y electrónica, brindando protección y confianza a sus clientes a fin de satisfacer sus necesidades. Trabajan continuamente por el desarrollo empresarial y el beneficio de las partes interesadas. La visión planteada es llegar a ser la empresa preferida por los clientes a nivel nacional, por sus servicios de seguridad integral, tecnología de punta, personal en continuo desarrollo profesional y una estructura financiera sólida.

En estos últimos años la empresa se ha visto afectada por el pago impuntual de algunos de sus clientes (en su mayoría del sector público), incluso, pese a que el servicio de vigilancia ha sido brindado en su totalidad quedan valores por cancelar de varios meses los cuales llegan a ser saldados hasta medio año después. Lo anteriormente expuesto genera un gran problema en la empresa al tener que recurrir al endeudamiento de manera reiterada en entidades financieras para cubrir sueldos de más de 100 empleados, cumplir con sus proveedores y con entidades de cumplimiento tributario y obligaciones patronales. Los valores de interés que se deben pagar a estas entidades por operaciones credi- 
ticias adquiridas son altos, lo que causa una pérdida de liquidez en la empresa. Así también, genera deficiencia en la rotación de capital, por lo cual no es posible ampliar el portafolio de servicios y productos.

Siendo las cosas así, el objetivo del presente trabajo de investigación consiste en diseñar un plan de gestión Administrativo Financiero dentro de la empresa SERSEIVI Cía. Ltda. el cual es muy importante ya que a través del mismo podemos identificar cuáles son los factores que inducen al alto endeudamiento y que afectan a la actividad económica de la empresa; así mismo verificar la existencia y correcta aplicación de procesos en el área administrativa y financiera.

\section{La gestión administrativa y financiera y su gran aporte en las empresas}

Los grandes avances tecnológicos, la fuerte competencia que existe en el mercado debido a la globalización y sobre todo los cambios continuos que tiene el comercio a nivel mundial han sido motivos más que suficientes para que en la actualidad las empresas, sin importar su tamaño y enfoque, se hayan visto en la necesidad de la implementación de una gestión administrativa - financiera, la cual permita alcanzar un nivel óptimo de desenvolvimiento en todas las áreas y recursos logrando así que exista el crecimiento esperado en todos los ámbitos.

Para lograr que la ejecución de la gestión administrativa - financiera se realice con éxito es necesario que la persona o grupo de personas que se encuentran a cargo tomen las decisiones acertadas para que la empresa pueda posicionarse por encima de sus competidores y lo más importante que importante que tenga proyección hacia el futuro. Chiavenato (2006) afirma:

La administración no es otra cosa que la dirección racional de las actividades de una organización, con o sin fines de lucro. Ella implica planeación, organización (estructura), dirección y control de las actividades realizadas en una organización, diferenciadas por la división del trabajo, ver Figura 1. Por tanto, la administración es imprescindible para la existencia, la supervivencia y el éxito de las organizaciones. (p.2) 


\section{Gestión administrativa}

Figura 1

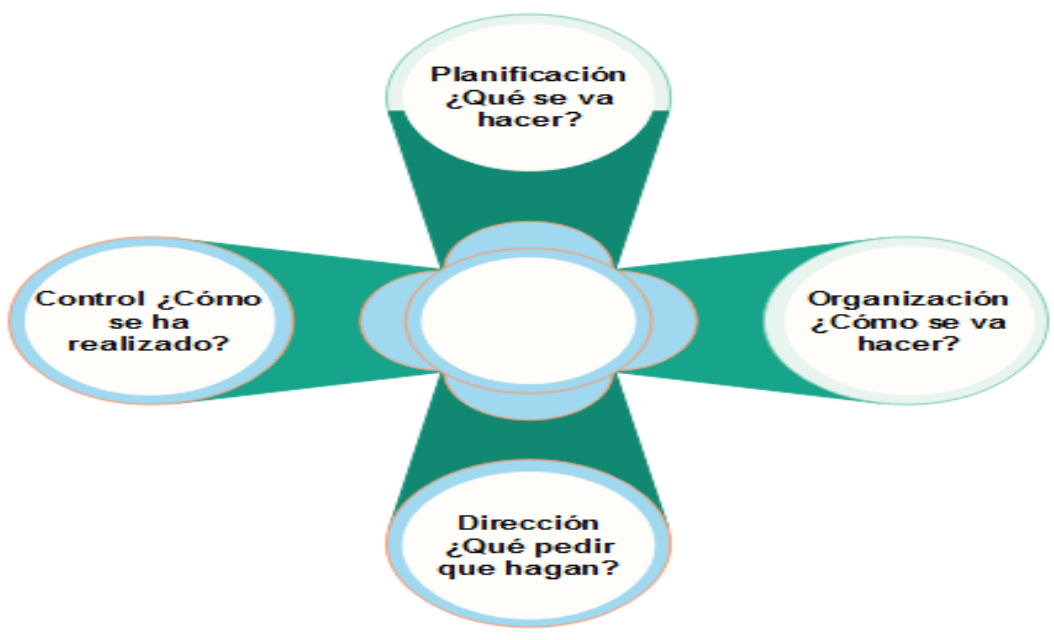

Fuente: Coulter, Robbins y Decenzo (2013)

En ese mismo contexto Münch (2010) indica que la gestión administrativa es el proceso más importante que toda empresa debe realizar desde el inicio de sus actividades ya que a través de su implementación y con la correcta coordinación de todos los recursos es posible conseguir la máxima productividad, calidad, eficacia, eficiencia y competitividad para de esta manera cumplir con los objetivos planteados por la organización.

Si bien es cierto que la gestión administrativa apoya en gran medida a las actividades que las empresas realizan diariamente, es conveniente que se emplee también un análisis del aspecto financiero, debido a que este recurso es uno de los más importantes dentro de cualquier organización. Por tal motivo, sus dirigentes están en la obligación de buscar alternativas que estén enfocadas en lograr rentabilidad y alcanzar una posición 
estratégica de liderazgo competitivo. Para poder lograr todos estos objetivos la gestión financiera es un pilar fundamental en el proceso de desarrollo, crecimiento y fortalecimiento de la empresa, así también es un apoyo al momento de tomar decisiones sobre aspectos financieros (De Ávila, Pérez y Vega, 2016).

La gestión financiera abarca varios procesos de gestión sin importar el tamaño o sector al que está enfocada la empresa, esto se logra a través de la integración de actividades y la optimización de las mismas. El objetivo de la gestión financiera es conseguir que los recursos económicos se manejen con eficiencia y eficacia para que de esta forma coadyuven a la difícil y compleja responsabilidad de la toma de decisiones (Cabrera, Fuentes y Cerezo, 2017).

Es muy importante que dentro de toda empresa se realice un seguimiento permanente y existan evaluaciones continuas sobre los resultados de la gestión financiera, saber de manera oportuna cuáles han sido los impactos de haber tomado una u otra decisión y en caso de que la misma necesite de alguna intervención o corrección poder hacerlo de inmediato sin afectar al rendimiento financiero de la organización.

Uno de estos modelos que en la actualidad es utilizado por un gran número de empresas es el Balanced Scorecard (BSC) el cual integra a todas las áreas de una empresa para poder cumplir con la visión y objetivos definidos en su plan estratégico.

La finalidad de la implementación de Balanced Scorecard es lograr alcanzar los objetivos planteados por los accionistas o empresarios, que el personal se sienta motivado para realizar su trabajo, que los clientes estén satisfechos con la atención y servicio recibido y acaparar clientes potenciales. Esta herramienta consiste en la implementación de indicadores de desempeño a través de los cuales se controlará y vigilará el correcto desempeño de operaciones y procesos ejecutados en la organización. Existen dos tipos de indicadores, los estratégicos que están alineados al plan estratégico y los secundarios que deben ser utilizados en las áreas cuyas actividades no afecten de forma directa en los procesos críticos para el negocio (Zamora y Álvarez, 2015). 
La Implementación del Balanced Scorecard o Cuadro de Mando Integral: Ayudará a los empresarios a que se controle de manera periódica el cumplimiento de las metas logrando así, que en caso de presentarse algún problema se tomen las medidas preventivas y lleguen a afectar significativamente a la empresa. La metodología empleada clasifica los objetivos en 4 perspectivas: Financiera, Clientes, Procesos internos y de Innovación y aprendizaje.

De acuerdo con este modelo, los objetivos, factores e indicadores se encuentran divididos en cuatro principales grupos interrelacionados, y cada uno de ellos representan diversas perspectivas de la empresa. Éstas son:

Financiera: ¿cómo debe aparecer la empresa ante sus accionistas/inversores para tener éxito financiero? Ha sido una de las perspectivas con mayor relevancia, ya que los objetivos se encuentran definidos de manera cuantificable. Uno de los objetivos principales es que la empresa alcance una gran rentabilidad, y es la perspectiva en la que se basan las otras tres para poder orientarse.

Procesos internos: ¿en qué procesos debe la empresa ser excelente para satisfacer a accionistas/inversores y clientes? Si la empresa no tiene definidos procesos para realizar actividades diarias, cubrir las necesidades de clientes o la de su cadena de valor, lo más probable es que no alcance los resultados esperados, al contrario, le costará pérdida de recursos de tiempo, económico y humano.

Innovación y aprendizaje: ¿cómo mantendrá y sustentará la empresa su capacidad de cambiar y mejorar, para conseguir lograr su misión? En esta perspectiva se definirán los objetivos en el ámbito del aprendizaje, crecimiento e innovación de la organización, y éstos serán el punto de partida para que se cumplan los objetivos planteados en las perspectivas anteriores. El Balanced Scorecard hace énfasis en la importancia que tiene la inversión de su personal y en la tecnología, ya que es a través de esto que se obtendrán resultados a largo plazo. (Jimenez, Erazo, Narváez y Quevedo, 2019)

Perspectiva del cliente: ¿cómo debe aparecer la empresa ante sus clientes para alcanzar su visión? Es aquí en donde se definen los objetivos que permiten a la organización 
mejorar la atención a sus clientes basándose en sus necesidades. En toda organización o empresa, se sabe que los clientes son la razón de ser de las mismas, por lo tanto, el tenerlos satisfechos y contar con su fidelidad, son objetivos claves para la organización. Todos los indicadores se encuentran relacionados entre sí a través de asociaciones causa-efecto que ponen en manifiesto la estrategia utilizada. Es importante saber que, para armar este cuadro de mando integral, los directivos deben tener definidos de manera clara la visión, misión de la empresa, a dónde quieren llegar y cómo lo quieren lograr (Kaplan y Norton, 1996).

\section{Endeudamiento y Apalancamiento}

La deuda y el apalancamiento son términos muy utilizados en las empresas y juegan un papel determinante en la formación y efectos de las crisis financieras. La deuda hace referencia a todos aquellos valores adicionales que un emisor acuerda pagar al inversor por un monto prestado, esto a través de cualquier mecanismo empleado. Por lo general al adquirir una deuda no se sabe a ciencia cierta el uso que se le da al dinero prestado, por lo cual se suele comparar con: otras fuentes de financiamiento y los activos disponibles que, de ser el caso, se utilizarían para pagarla. Es esta medición la que justamente origina el concepto de apalancamiento (Vargas y Díaz, 2017).

El apalancamiento se refiere a cuál es el grado en el que la empresa hace uso de la deuda para poder financiar sus actividades, en resumen, no es más que utilizar el dinero prestado para financiar las operaciones diarias de la compañía, el dinero no va a provenir de fondos propios o del aporte de sus accionistas, sino más bien se lo adquirirá mediante un crédito. El aumento de la deuda por parte de la empresa o del apalancamiento financiero va a repercutir en la rentabilidad, que obviamente depende del costo financiero de la deuda adquirida. El apalancamiento tiene entre sus ventajas aumentar significativamente la rentabilidad de la empresa, pero entre sus desventajas es la de generar iliquidez en la empresa consecuencia del fracaso de su operación (Salazar y Bajaña, 2017). 
Razón de Endeudamiento: Es el grado en el que los activos de la empresa han sido financiados a través de deudas a corto y largo plazo. Este rubro debe estar cercano el $50 \%$ lo que quiere decir que la mitad de los medios deben ser financiados haciendo uso de la deuda y la otra mitad con fuentes propias, lo recomendable es que este equilibrio se mantenga (García, Camacho y Velázquez, 2017). Se calcula de la siguiente manera:

$$
\text { Razón de endeudamiento }=\frac{\text { Pasivo total }}{\text { Activo total }}
$$

Razón de Apalancamiento: Es la relación existente entre el pasivo total y el patrimonio total que posee la empresa. Se calcula de la siguiente manera:

$$
\text { Razón de apalancamiento }=\frac{\text { Pasivo total }}{\text { Patrimonio total }}
$$

\section{Indicadores de endeudamiento}

Sirven para poder analizar la cantidad y la calidad de la deuda de la empresa, y a su vez concluir qué tan estable es su estructura financiera. Algunos de los indicadores existentes son (Cuenca, 2014):

Ratio de endeudamiento a largo plazo: Es la relación existente entre el Pasivo no corriente y el Patrimonio neto.

$$
\begin{aligned}
& \text { Razón de endeudamiento } L / P \\
& =\frac{\text { Pasivo no corriente }}{\text { Patrimonio neto }}
\end{aligned}
$$

Ratio de endeudamiento a corto plazo: Es la relación existente entre el Pasivo corriente y el Patrimonio neto.

$$
\text { Razón de endeudamiento } C / P=\frac{\text { Pasivo corriente }}{\text { Patrimonio neto }}
$$


Ratio de autonomía: es lo contrario al ratio de endeudamiento total, es decir muestra la cantidad de recursos propios que tiene la empresa para cubrir deudas.

$$
=\frac{\begin{array}{c}
\text { Razón de autonomía } \\
\text { Patrimonio neto }
\end{array}}{\text { (Pasivo no corriente }+ \text { Pasivo corriente })}
$$

Ratio de garantía: Hace referencia a la seguridad que tienen los acreedores de cobrar su deuda y es recomendable que este valor se encuentre entre 1,5 y 2,5. Mientras más pequeño sea el valor quiere decir que la empresa va a tener cada vez más dificultades de poder cubrir sus deudas y está más cerca de la quiebra.

Razón de garantía

$$
=\frac{\text { Activo }}{\text { (Pasivo no corriente }+ \text { Pasivo corriente })}
$$

Ratio de calidad de la deuda: Es la relación de las deudas a corto plazo con las deudas a largo plazo, mientras más bajo es el resultado mayor será la capacidad de que la empresa pague las deudas tomando como referencia los plazos.

$$
=\frac{\begin{array}{c}
\text { Ratio calidad de deuda } \\
\text { Pasivo corriente }
\end{array}}{\text { (Pasivo no corriente }+ \text { Pasivo corriente })}
$$


Estos indicadores son muy importantes ya que pone en manifiesto los cambios o ajustes que se debe realizar para que el margen de rentabilidad no se vea perjudicado y que la empresa no se vea afectada por el alto endeudamiento. Algunos de los indicadores que también sobresalen, además de los ya citados anteriormente, son (López y Sierra, 2015):

Endeudamiento financiero: Porción de activo de la empresa que está financiado por obligaciones financieras.

$$
\begin{aligned}
& \text { Endeudamiento financiero } \\
& =\frac{\text { obligaciones finaniera }}{\text { Activo total }}
\end{aligned}
$$

Impacto de la carga financiera: Porcentaje de gastos financieros en relación a los ingresos de la institución.

$$
\begin{aligned}
& \text { Impacto de carga financiera } \\
& =\frac{\text { Gastos financieros }}{\text { Ingresos }}
\end{aligned}
$$

Índice de solvencia: Mide la capacitada de la empresa para hacer frente al pago de todas sus deudas y obligaciones.

$$
\text { Índice de solvencia }=\frac{\text { Activo total }}{\text { Pasivo total }}
$$

\section{METODOLOGÍA}

El tipo de investigación que se empleó en la elaboración del presente trabajo es no experimental, debido a que no se manipularon las variables.

El enfoque aplicado es cualitativo a través de la investigación exploratoria, es decir se recopiló datos aplicando diferentes técnicas como cuestionarios, observaciones, entrevistas, etc. con la finalidad de llevar a cabo un análisis más amplio y lograr una mejor comprensión de situaciones. Basado en los resultados obtenidos en el desarrollo de este 
trabajo se aplicó el enfoque cuantitativo ya que fue necesario la cuantificación de los resultados tomando en consideración las observaciones.

En virtud de lo antes mencionado se aplicó el nivel de investigación Descriptivo-Explicativo puesto que se analizó las causas que originan la problemática del endeudamiento.

Se utilizaron los métodos Inductivo-Deductivo para recolectar información a través de entrevistas no estructuradas al personal administrativo-financiero, con lo cual se establecen generalizaciones a partir de lo común en varios casos.

Así también se aplicó el método histórico-lógico con la finalidad de poder analizar los antecedentes, las causas y las condiciones en que se ha desarrollado el análisis de la gestión administrativa-financiera a través de los años.

Del mismo modo se utilizó el método estadístico para poder analizar la información recolectada en ambas variables, esto a través del manejo de datos cualitativos y cuantitativos obtenidos en la investigación.

Se aplicó también el método analítico-sintético a través del cual facilitó el análisis y la clasificación de las fuentes de información recopiladas en busca de la esencia de las ideas.

Se utilizaron 4 técnicas (Tabla 3) para la recolección de datos, ellas son:

Técnicas e instrumentos de investigación

Tabla 3

\begin{tabular}{|c|c|c|c|}
\hline Técnica & Instrumento & Personal & Lugar \\
\hline Encuesta & Cuestionario & Administrativo & Empresa \\
\hline Entrevista & Guía de preguntas & $\begin{array}{l}\text { Gerente General y } \\
\text { Contadora }\end{array}$ & Empresa \\
\hline $\begin{array}{l}\text { Análisis docu- } \\
\text { mental }\end{array}$ & Ficha de contenido & Administrativo & $\begin{array}{l}\text { Lugar de tra- } \\
\text { bajo }\end{array}$ \\
\hline Focus group & Guía discusión grupal & $\begin{array}{l}\text { Administra- } \\
\text { tivo/Operativo } \\
\text { (guardias) }\end{array}$ & Empresa \\
\hline
\end{tabular}

Fuente: Sampieri (2014) 
La Población estuvo conformada por el personal administrativo y operativo que labora en la empresa Servicios de Seguridad y Vigilancia SERSEIVI Cía. Ltda. con un total de 51 personas (Tabla 4). Para el presente trabajo no se utilizó muestreo, puesto que se trabajó con todo el universo.

\section{Universo de estudio}

Tabla 4

\begin{tabular}{|c|c|c|c|}
\hline Cargos & Población & Técnica & Instrumento \\
\hline \multirow{2}{*}{$\begin{array}{l}\text { Gerente } \\
\text { General }\end{array}$} & \multirow{2}{*}{1} & Entrevista/ & \multirow{2}{*}{$\begin{array}{l}\text { Guía de preguntas/guía } \\
\text { discusión grupal }\end{array}$} \\
\hline & & Focus group & \\
\hline \multirow{2}{*}{ Contadora } & \multirow{2}{*}{1} & Entrevista/ & \multirow{2}{*}{$\begin{array}{l}\text { Guía de preguntas/guía } \\
\text { discusión grupal }\end{array}$} \\
\hline & & Focus group & \\
\hline \multirow{2}{*}{ Secretaría } & \multirow{2}{*}{1} & Encuesta/ & \multirow{2}{*}{$\begin{array}{l}\text { Cuestionario/guía discu- } \\
\text { sión grupal }\end{array}$} \\
\hline & & Focus group & \\
\hline \multirow{2}{*}{$\begin{array}{l}\text { Auxiliar } \\
\text { contable }\end{array}$} & \multirow{2}{*}{1} & Encuesta/ & \multirow{2}{*}{$\begin{array}{l}\text { Cuestionario/guía discu- } \\
\text { sión grupal }\end{array}$} \\
\hline & & Focus group & \\
\hline \multirow{2}{*}{$\begin{array}{l}\text { Jefe } \\
\text { Operaciones }\end{array}$} & \multirow{2}{*}{1} & Encuesta/ & \multirow{2}{*}{$\begin{array}{l}\text { Cuestionario/guía discu- } \\
\text { sión grupal }\end{array}$} \\
\hline & & Focus group & \\
\hline $\begin{array}{l}\text { Supervisor/ } \\
\text { Guardias Seguridad }\end{array}$ & 46 & Focus group & Guía discusión grupal \\
\hline
\end{tabular}

Fuente: SERSEIVI Cía. Ltda. (2019)

\section{RESULTADOS}

Una vez que todas las encuestas fueron contestadas se realizó el análisis pertinente, especificando todas las preguntas con sus respectivas variables, el número de personas y el porcentaje para cada respuesta. Así también, los resultados se encuentran plasmados en una tabla o gráfico para una mejor interpretación.

\section{Razón de endeudamiento total}

Este índice permite conocer el nivel de endeudamiento que posee la empresa respecto a los acreedores sobre sus activos. En el año 2017 por cada dólar que posee la empresa 
USD. 0,63 debe a los acreedores. Este valor disminuye en el año 2018 a USD. 0,35. Con estos resultados se determina que en el año 2017 del 100\% de los activos con los que contaba SERSEIVI Cía. Ltda. el 63\% les pertenecía a los acreedores.

Los resultados de las entrevistas realizadas tanto al Gerente General como a su Contadora corroboran el valor del nivel de endeudamiento para el año 2018, esto es debido a que en este último período la empresa no ha tenido que recurrir a grandes deudas en entidades financieras puesto que han aplicado la re-inversión de las Utilidades generadas o la venta de activos que ya eran obsoletos.

Su contadora señala que por lo general recurren al endeudamiento a corto plazo pues es más conveniente para solventar gastos corrientes. Basándonos en el análisis horizontal realizado entre los períodos 2017 y 2018 podemos determinar que la Calidad de Deuda a corto plazo que ha mantenido la empresa, por cada UDS. 1,00 de deuda que posee con sus acreedores, USD. 0,98 y USD. 1,00 tienen vencimiento corriente para el año 2017 y 2018 respectivamente.

Por otra parte, tomando como referencia el análisis horizontal, es evidente el alto nivel de endeudamiento de la empresa en el período del 2017, llegando a cancelar USD. 33109,20 únicamente en intereses. Este rubro disminuye en el año 2018 en un 34,52\%, cancelando la cifra de USD. 21680,02.

El principal motivo para que la empresa acuda a los préstamos en entidades financieras es debido a la falta de pago en los tiempos establecidos por parte de sus clientes, la mayoría de ellos del sector público, y la causa es atribuida, en un 60,00\%, a la falta de recursos por parte del Estado.

\section{Apalancamiento financiero}

La empresa ha tenido un apalancamiento financiero de 1,71 en el año 2017, mientras que en el año 2018 disminuyó en un 69 \% con un índice de 0,53, lo que significa que en el primer período de análisis estaba sobre endeudada, es decir, estaba empleando más deuda para financiar sus activos y obtener utilidades. 
El índice obtenido en el año 2018 muestra que el 53\% de los recursos con los que opera la empresa pertenece a los acreedores; el resto es aportado por los socios. En este sentido, se interpreta que por cada USD. 0,53 de pasivos se cuenta con USD. 1,00 de activos para financiarla.

\section{Impacto de carga financiera}

El valor de la carga financiera indica que del total de ventas que ha realizado la empresa durante su período hay que destinar cierto porcentaje para pagar los gastos financieros. Al observar los resultados del análisis horizontal se determina que la carga financiera fue superior en el año 2017 comparada con el año 2018, en el primer año el índice fue de 0,023, mientras que en este último año disminuyó en un 52,15\%, llegando a un índice de 0,011 .

Haciendo uso del análisis vertical en cada período se establece que para el año 2017 los gastos financieros representaban el $2,43 \%$ del total de egresos, mientras que para el año 2018 su porcentaje fue de $1,14 \%$.

\section{Índice de solvencia}

El ratio indica cuántos dólares tiene la empresa, entre bienes y derechos, por cada dólar que tiene de deuda. SERSEIVI Cía. Ltda. ha tenido un ligero incremento de su solvencia este último año con un valor de 2,88; mientras que, en el 2017 éste era de 1,58, esto quiere decir que la empresa cuenta en su activo con USD. 2,88 por cada dólar que tiene de deuda. Este incremento es debido al aumento en los activos y disminución de los pasivos en el último período.

El valor óptimo para este ratio está en torno a 1,50, si consideramos los resultados de los dos años en cuestión, se deduce que actualmente la posición de los acreedores se encuentra fortalecida, pero, sin embargo, demuestra que hay un exceso de activos improductivos. 


\section{Ratio de autonomía}

La empresa en el año 2017 obtuvo un índice de 0,58 y en el año 2018 su valor subió a 1,89 , lo que significa que es menos dependiente de sus acreedores. El valor óptimo que se da para índice es de mínimo 0,7, y en los resultados obtenidos se deduce que la autonomía financiera de la empresa en el año 2017 estaba por debajo de lo recomendado, en resumen, no gozaba de estabilidad financiera pero aun así la empresa nunca ha estado al borde la quiebra. Es evidente que durante el año 2018 ha existido una mejora considerable en este índice, siendo más del doble del obtenido el año anterior, esto gracias al aumento del patrimonio y disminución del pasivo de la empresa; pero pese a esto no hay que dejar de analizar el resto de indicadores los cuales serán de gran ayuda en el momento de tomar decisiones y ejecutar planes de acción. Los resultados de los diversos índices de endeudamiento que se obtuvo en la empresa en los períodos 2017 y 2018 se presentan en la Figura 2: 


\section{Indicadores}

Figura 2

\section{Indicadores de endeudamiento}
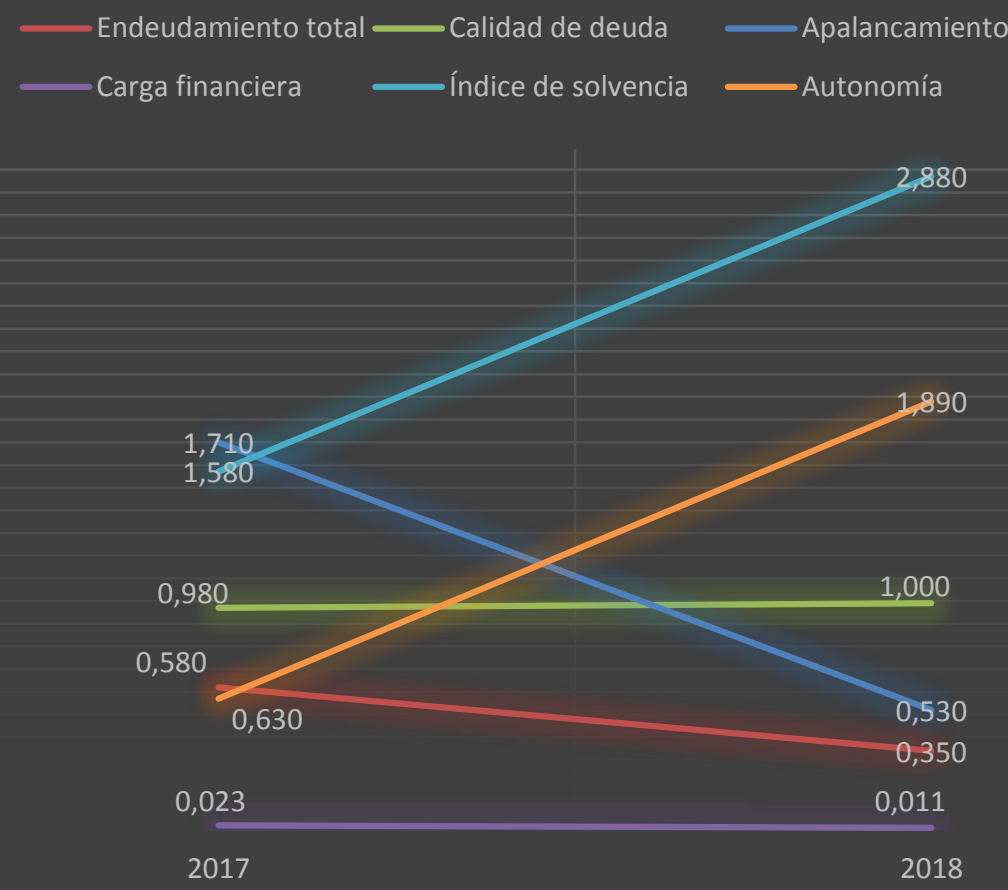

El contador también indica que la empresa no cuenta con indicadores que pongan en manifiesto su solvencia, únicamente este valor está plasmado en un documento el cual es utilizado para procesos de contrataciones, pero no se realiza ningún análisis o planes de acción basados en el mismo.

Para el año 2017 los gastos operacionales representaban el 93,16\% del Total de Ventas (Figura 3), mientras que en el año 2018 el porcentaje fue de $94,26 \%$, lo que se traduce que en este último período la empresa tuvo un ligero incremento en este rubro. 


\section{Ventas vs. Gastos operacionales}

Figura 3
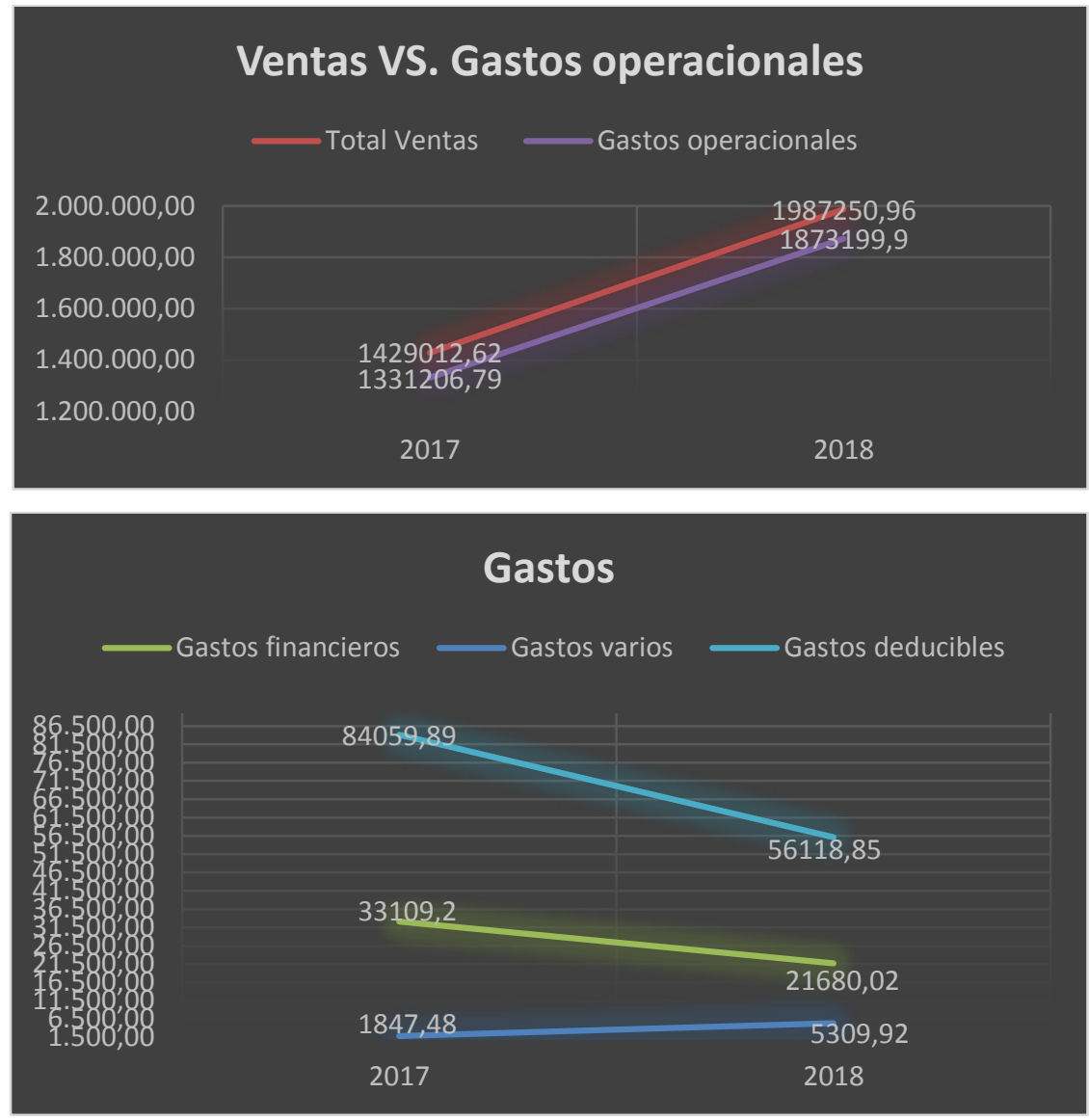

Figura 4. Gastos

Importa, y por muchas razones, la manera en cómo la empresa maneja el control de gastos, no sólo el de financieros sino también los generales. Por ejemplo, el rubro de Gastos Varios (Figura 4), en el año 2017 fue de un monto de USD. 1847,48, en cambio al revisar la cifra correspondiente al año 2018 este valor se incrementa hasta los USD. 5309,92 , aumentando en un $187,41 \%$. Es necesario analizar qué factores fueron los que provocaron que esta cuenta haya incrementado en más del $100 \%$.

En este sentido se comprende que la totalidad de gastos operacionales efectuados en el último período analizado es superior al del año 2017; en cambio, los gastos no operaciones disminuyeron este último año en comparación con el año anterior. 


\section{Procesos administrativos}

La empresa suele definir cuáles serán los objetivos a alcanzar, tarea que es realizada por el Gerente General con el apoyo del área Contable tomando en cuenta los resultados de un período y basándose en distintas pautas y procedimientos que son analizados en conjunto.

Cuando se realiza la planeación estratégica, la empresa no involucra a todo el personal administrativo y operativo, dejando a un lado a otras áreas que también son importantes en el giro del negocio y que podrían aportar con recomendaciones o sugerencias de gran valor. El $60,00 \%$ de los encuestados indicaron que casi siempre participan en la planificación de los procesos administrativos que realiza la institución.

Ahora bien, todo lo acordado en el levantamiento de estos procesos no es puesto a conocimiento oportuno de los empleados, tal es el caso del plan estratégico definido; el cual, pese a que es ejecutado, no es difundido de manera efectiva a todas las áreas de la empresa.

Con respecto a las actividades que son realizadas por los empleados, se determina que en la empresa existe un orgánico funcional, en el cual se describen a detalle todas las actividades y responsabilidades de cada área o departamento, pero el mismo no se encuentra actualizado o no está al alcance de todos para su conocimiento.

La percepción en general es que casi todo el personal administrativo se encuentra capacitado para realizar las actividades encomendadas, obteniendo como resultado mayoritario que saben "Lo suficiente" para ejecutarlas.

Existe una buena coordinación de las actividades entre las distintas áreas ya que el $100,00 \%$ de los encuestados así lo manifiestan, siendo beneficioso, por una parte, el hecho que no son muchas personas las que trabajan en oficinas por lo tanto la comunicación es fluida y rápida. Aquellas notificaciones o partes que deben dar a conocer al personal de guardias son transmitidas a través del supervisor, quien, de ser el caso, les hace firmar un documento en constancia que tienen conocimiento de cierto asunto. Los resultados obtenidos en la encuesta son los siguientes. 
Pero si se analiza este resultado con el obtenido en la difusión del plan estratégico existe una discordancia, estos resultados revelan que la comunicación es más fluida entre los empleados en actividades del día a día y no en aquellas tales como planes y estrategias que son cruciales para el negocio a largo plazo.

Los empleados del área administrativa manifiestan que están satisfechos en un 100,00\% con el liderazgo por parte de la Gerencia General, señalan que es un factor importante para que todos puedan cumplir con sus funciones a pesar de los inconvenientes presentados; pero, la motivación se ve afectada en ocasiones debido a factores externos a la empresa, específicamente por la impuntualidad en sus remuneraciones, que por política de la empresa debería ser siempre de manera oportuna para lograr así un óptimo rendimiento por parte del personal operativo y administrativo.

\section{Sistema de control}

Se evidencia que la empresa no lleva un control de marcación o bitácora para el registro de entrada o salida de la oficina. Por otra parte, el personal de guardias, al cumplir con sus funciones en distintos lugares reportan su asistencia a través de radio hacia la central en donde registran su hora de ingreso, atrasos o faltas en caso de existir. Sin embargo, existen falencias en el reporte que deben hacerlo cada hora o dos horas, de acuerdo a lo especificado para el punto en el que se encuentran, aduciendo que estaban ocupados o simplemente se les olvidó.

Es también relevante señalar que el desempeño dentro de la empresa por parte del personal administrativo no es evaluado periódicamente, esto se evidencia en los resultados obtenidos en la encuesta en donde el $80,00 \%$ señala que se lo realiza "A veces" y el $20,00 \%$ que nunca lo ha hecho.

Resulta así mismo interesante saber que el $60,00 \%$ de los encuestados manifestaron que tienen reuniones "Casi siempre" para poder definir metas, o dar seguimiento a actividades o temas que requieren de un control continuo, mientras que el 40,00 \% señaló que lo hacen "A veces". 


\section{Control presupuestario}

El $60,00 \%$ de los encuestados manifiesta que es "Realmente importante" el contar con estrategias dentro de la empresa para la asignación adecuada de recursos, mientras que un $40,00 \%$ señala que es "Muy importante". Por otra parte, de las 5 áreas incluidas en el análisis, el 60,00\% de los encuestados está "Totalmente" de acuerdo en la manera en la que se distribuyen los recursos para los Supervisores, siendo así el puntaje más alto; y, un $40,00 \%$ para las áreas restantes. Mientras que, con un empate del $40,00 \%$ los encuestados señalaron que los recursos asignados tanto para el área Contable como el Jefe de Operaciones son "Medianamente" eficientes

Es también relevante el hecho de que la mayoría de personas que participaron en el Focus group señalaron que es necesario que la empresa haga inversión en recursos tecnológicos, equipos de rastreo, cámaras de seguridad de última generación, sistema de monitoreo de mejores características.

De igual manera, manifiestan la importancia de la publicidad que tiene que realizar la empresa, sea a través de medios convencionales o digitales, la cual ha sido dejada a un lado desde hace mucho tiempo.

\section{Administración de inventarios}

La empresa utiliza la herramienta Excel para el manejo de inventarios, y no a través de un sistema informático especializado, que sería lo más conveniente. Tomando como referencia los resultados de la encuesta, un $60,00 \%$ señala que el inventario está "Actualizado" y un 40,00\% que "No lo está". Al haber esta contradicción entre los encuestados, sólo pone en evidencia que los inventarios no están siendo llevados de manera correcta y que cuando han solicitado información al día, el responsable del área no lo ha tenido.

\section{Estados financieros}

La empresa tiene como buena práctica revisar periódicamente datos de los ingresos, egresos, gastos, ventas y analizar posibles problemas futuros; pero su análisis no es muy 
profundo, toman como referencia sólo algunos campos del balance general y de resultados y no otros factores que son críticos al momento de la toma de decisiones. Los resultados financieros son analizados por auditores internos y externos siguiendo la normativa establecida, así también se determina que esta información es solicitada por Gerencia General al área contable para la toma de decisiones y planeación estratégica. Los directivos de la empresa no han implementado un plan de inversión desde el inicio de sus actividades, esto lo manejan obteniendo estimados de cuánto utilizarán como reinversión con la finalidad de repotenciar su dotación de armamento y equipo nuevo de trabajo.

\section{Presupuestos}

Existen criterios divididos en las respuestas de entrevistas realizadas, por un lado, el Gerente General indica que antes de iniciar el período se elabora un presupuesto anual y es en base a esta información que en el transcurso de ese año se procede con los respectivos planes de financiamiento; no obstante, el Contador de la empresa manifiesta que no se evalúa la gestión presupuestaria, o al menos para realizarla el área contable no es tomada en cuenta.

\section{Clientes}

Del total de encuestados un $40 \%$ manifiesta que se realizan entrevistas o encuestas a los clientes, públicos o privados, para poder medir el grado de satisfacción del servicio entrego. Un $40 \%$ indica que no se realiza dicho seguimiento; y el $20 \%$ restante dice no tener conocimiento al respecto. Dentro de este marco, y con resultados más favorables, se pone en evidencia que el $80 \%$ de los encuestados indican que las novedades, quejas o inconformidades que reportan los clientes sí son atendidas, es decir, se escala al área correspondiente, se analizan causas, consecuencias, y se toman las medidas correctivas lo antes posible. 


\section{Financiero}

Al realizar el análisis de este elemento, se determina la gran importancia de la implementación de KPl's financieros, llegando a la conclusión de que los mismos son imprescindibles en cualquier organización desde el momento de su creación. Pero el uso de estos mecanismos de evaluación de la gestión no es únicamente requerido en el área financiera, sino también en cada una de las áreas en donde la organización requiere que los objetivos planteados sean medidos con cierta frecuencia. Además, se definirá quién o quiénes son los responsables de la medición de cada indicador, así también quiénes serán los usuarios interesados en cada uno de ellos. En la Figura 5 se plantea un registro en el cual la empresa puede llevar el control de cada indicador establecido, considerando que, mientras más indicadores se tengan va a volverse más difícil su interpretación.

\section{Formato registro KPI}

\section{Figura 5}

\begin{tabular}{|c|c|c|c|c|c|c|c|c|c|c|c|c|c|c|c|}
\hline \multirow{2}{*}{ ÁREA } & \multirow{2}{*}{ RESPONSABLE } & \multirow{2}{*}{ OBJETIVO } & \multirow{2}{*}{ INDICADOR } & \multicolumn{12}{|c|}{ RESULTADOS AÑO: } \\
\hline & & & & Ene & Feb & Mar & $A b r$ & May & Jun & Jul & Ago & Set & Oct & Nov & Dic \\
\hline & & & Razón de endeudamiento & & & & & & & & & & & & \\
\hline & & & Razón de apalancamiento & & & & & & & & & & & & \\
\hline & & & Calidad de deuda & & & & & & & & & & & & \\
\hline & & & Carga financiera & & & & & & & & & & & & \\
\hline & & & Solvencia & & & & & & & & & & & & \\
\hline
\end{tabular}

En base al estado de resultados, es posible realizar la comparación de los años 2017 y 2018 para cada cuenta a través del análisis Horizontal y Vertical, lo cual representa una herramienta muy útil y rápida al momento de querer analizar más de un período, identificar sus aciertos y falencias, y establecer medidas correctivas urgentes de ser el caso (Tabla 5). 


\section{Análisis horizontal}

Tabla 5

\begin{tabular}{|l|l|l|l|l|l|}
\hline \multicolumn{9}{|c|}{ ANÁLISIS HORIZONTAL } & AÑ̃ 1 & AÑ 2 & VARIACIÓN & \% \\
\hline \multicolumn{1}{|c|}{ CÓDIGO } & \multicolumn{1}{|c|}{ DETALE } & & & & \\
\hline $\mathbf{1}$ & Activo & & & & \\
\hline 11 & Correiente & & & & \\
\hline 113 & Disponible & & & & \\
\hline 114 & Exigible & & & & \\
\hline 12 & Otros Corrientes & & & & \\
\hline 121 & Fijo & & & & \\
\hline 122 & Propiedades & & & & \\
\hline $\mathbf{2}$ & Depreciación Acumulada & & & & \\
\hline 21 & Pasivo & & & & \\
\hline 211 & Corriente & & & & \\
\hline 212 & Cuentas y documentos por pagar & & & & \\
\hline 213 & Obligaciones bancarias & & & & \\
\hline
\end{tabular}

Se determina que la empresa no prepara un plan de inversión y presupuesto antes de iniciar un nuevo período, lo que ha traído algunos problemas en temas logísticos y financieros en el transcurso del año. Se propone que cada período la Gerencia General junto con el área de Contabilidad preparen el plan de inversión y destinen un presupuesto real, tomando en cuenta gastos en aspectos logísticos, legales, inversiones como tal, etc. Por último, es necesaria la elaboración de estrategias de gestión y recuperación de cartera, puesto que la falta de pago de los clientes es la causa principal de su endeudamiento, la persona encargada de esta función podrá hacer uso del formato que se visualiza en la Figura 6. 


\section{Formato registro gestión cartera}

Figura 6

ANÁLISIS DE CARTERA CLIENTES SERSEIVI

\begin{tabular}{|c|c|c|c|c|c|c|c|c|c|c|c|}
\hline \multicolumn{12}{|c|}{ FECHA DE CORTE: } \\
\hline \multirow{3}{*}{ Cuenta } & \multirow{3}{*}{ CONCEPTO } & \multirow{3}{*}{$\begin{array}{c}\text { Saldo } \\
\text { Trimestre }\end{array}$} & \multirow{3}{*}{$\begin{array}{l}\text { Valor } \\
\text { Inicial }\end{array}$} & \multicolumn{6}{|c|}{ CLASIFICACION } & \multirow{3}{*}{ TOTAL } & \multirow{3}{*}{ OBSERVACIONES } \\
\hline & & & & 0-30 Días & \multirow{2}{*}{\begin{tabular}{|c|} 
Valor \\
\end{tabular}} & \multirow{2}{*}{\begin{tabular}{|c|} 
61-90 Días \\
Valor
\end{tabular}} & \multirow{2}{*}{$\begin{array}{c}\begin{array}{c}91-180 \\
\text { Dias }\end{array} \\
\text { Valor }\end{array}$} & \multirow{2}{*}{\begin{tabular}{|c} 
181-360 Días \\
Valor
\end{tabular}} & \multirow{2}{*}{$\begin{array}{c}\begin{array}{c}\text { Más de } 360 \\
\text { Días }\end{array} \\
\text { Valor }\end{array}$} & & \\
\hline & & & & Valor & & & & & & & \\
\hline & & & & & & & & & & & \\
\hline & & & & & & & & & & & \\
\hline & & & & & & & & & & & \\
\hline & & & & & & & & & & & \\
\hline & & & & & & & & & & & \\
\hline
\end{tabular}

\section{Procesos internos}

La empresa cuenta con la certificación ISO 9001:2015 para lo cual ha levantado varios procesos con sus respectivos registros, instructivos, normas y especificaciones. Si bien es cierto que toda esta documentación está en regla, con las revisiones del caso, el problema es que no todas las áreas tienen conocimiento de los procesos de los que son responsables. Por ejemplo, en el área de Logística, pese a que existe un proceso y el mismo ha sido aprobado por Auditoría, el jefe responsable del área no hace uso del mismo, lo que conlleva a que los indicadores no se reporten de manera correcta y la información no sea verdadera. Para mitigar este problema, se plantea socializar a toda la organización el Plan del Sistema Integrado de Gestión, indicando procesos, responsables, procedimientos, registros y sus indicadores (Figura 7). 
Revista Arbitrada Interdisciplinaria KOINONIA

Año IV. Vol IV. N ${ }^{\circ}$. Julio - Diciembre 2019

Hecho el depósito de Ley: FA2016000010 ISSN: 2542-3088

FUNDACIÓN KOINONIA (F.K). Santa Ana de Coro. Venezuela.

Jorge Enrique Sánchez; José Luis Sánchez Espinosa; Jorge Hernando Sánchez Espinosa; Verónica Alexandra Sánchez Espinosa

\section{Registro procesos}

Figura 7

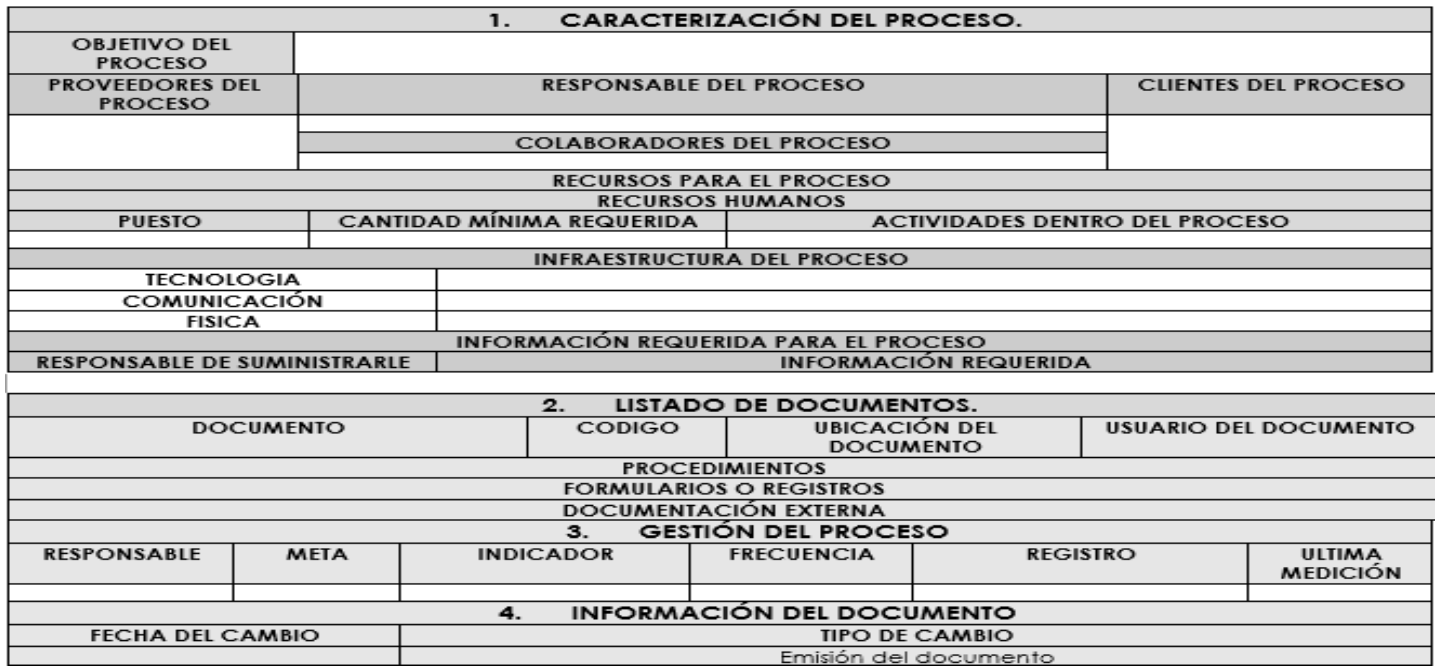

Realizar una evaluación de procesos con frecuencia determinada y en base a los resultados establecer planes de mejora, el encargado de dirigir esta tarea será asignado por el Gerente General. Así también, en el caso de que algún proceso actual se encuentre desactualizado o haya la posibilidad de realizar posibles mejoras, se solicitará a los involucrados que procedan a llenar el registro de Solicitud de Acción (Tabla 6).

\section{Solicitud de acción}

\section{Tabla 6}

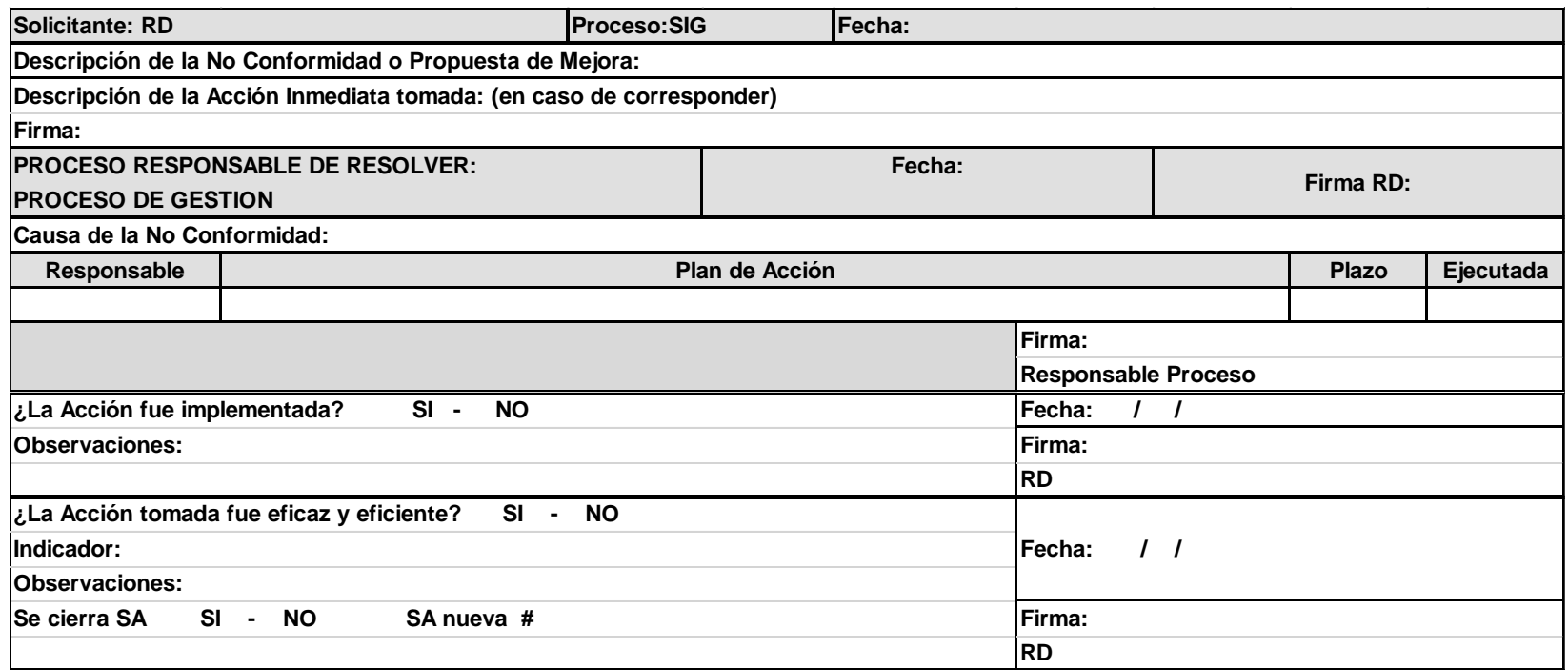




\section{Clientes}

Es indispensable que la organización cuente con personal especializado en el área de comercialización/marketing, de esta manera crearán estrategias para llegar a más clientes, sobretodo del sector privado que es lo que le conviene. El marketing puede ser tradicional o digital, es decir algo más innovador a través de redes sociales, páginas web, etc. Dentro de este orden de ideas, es muy importante medir la satisfacción del cliente respecto el servicio brindado, lo cual es posible realizarlo a través de una encuesta de satisfacción (Figura 8), con cierto período de frecuencia para no llegar a incomodar al cliente, logrando así la fidelidad de su parte y afianzar la relación ya existente. Aprovechar el hecho de contar con la certificación ISO 90001-2015 y dar a conocer a clientes las ventajas que la empresa posee con esta implementación, normas que cumple, estándares a seguir, calidad en sus procesos o el hecho de brindar mayor valor a los clientes con un reducido impacto ambiental. 


\section{Formato registro encuesta satisfacción cliente Figura 8}

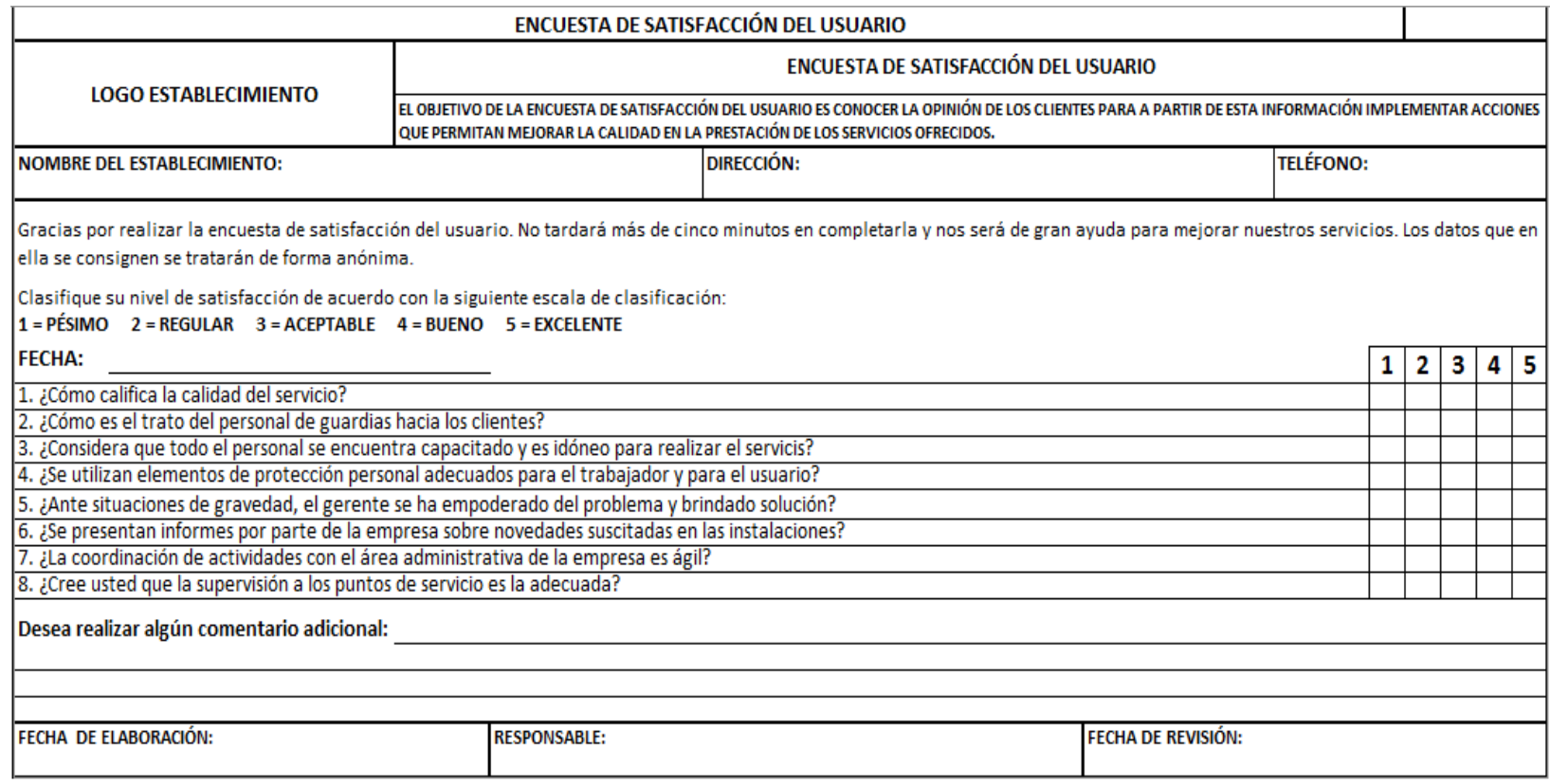

\section{Aprendizaje, TTHH y TI}

La empresa debe incluir en su presupuesto anual el ampliar su portafolio de servicios, enfocándose también en aquellos que hacen uso de la tecnología, algo que sea novedoso, llame la atención del cliente y sobretodo cumpla con sus espectativas. Debe fortalecer el servicio que posee actualmente de alarmas y monitoreo, acaparando más clientes no sólo en la zona de Cuenca. El área de Talento Humano también está incluida en este apartado ya que debe establecer un correcto proceso de reclutamiento y selección de personal, así también el desarrollo de competencias, su medición, evaluaciones y seguimiento. Todo el personal del área administrativa necesita capacitación en ofimática, ya que se evidencia que todos ellos, sin excepción hacen uso de esta herramienta. Se requiere que la persona del área de secretaría se especialice en el manejo del portal de compras pública, no sólo como proveedor sino también como entidad contratante, esto con la finalidad de que puedan entender cuál es su visión en el momento de revisar o calificar propuestas enviadas. Debe establecerse un plan de 
incentivo para el personal a través del cumplimiento de metas, por ejemplo puede ser haciendo uso de los indicadores ya planteados en los procesos de cada área. Tomar en cuenta un factor importante, que el área de $\mathrm{RRHH}$ debe coordinar con Gerencia General y Contabilidad para la asignación de un presupuesto con el cual podrá realizar todas las actividades anteriormente planteadas.

\section{Discusión}

La empresa no cuenta con una herramienta financiera que sea capaz de realizar análisis eficientes haciendo uso de indicadores financieros para de esta manera poder emitir información confiable para sus directivos.

En el año 2017 la empresa alcanzó un alto nivel de sobre endeudamiento debido a la gran cantidad de créditos que fueron solicitados tanto a cooperativas como a bancos en la ciudad. Empezó a adquirir más deuda con la finalidad de poder financiar sus activos y así obtener utilidades.

Las decisiones que ha tomado la Gerencia General lo ha realizado basándose en resultados del balance general al final de cada período, analizando los resultados obtenidos, pero por ejemplo, no se realiza un análisis vertical y horizontal para los períodos consecutivos y determinar así el aumento, disminución o variación de las diversas cuentas de un período a otro.

El índice de solvencia que alcanzó la empresa en el año 2018 es mucho más alto que el recomendado establecido, por un lado, esto puede demostrar que la situación económica de la empresa está fortalecida en comparación con el año 2017, pero, sin embargo, esto delata que existe un exceso sumamente alto de activos que son improductivos, lo que a la final no contribuye en nada positivo en la economía de la empresa.

Es evidente que en la empresa existen varios procesos administrativos-financieros que no están siendo llevados de manera correcta o simplemente no ha habido desde la creación de la misma. Es necesario revisar planes estratégicos definidos, orgánico, metas y objetivos planteados, así también actualizar planes que hoy en día están obsoletos, 
todo esto acompañado del levantamiento de indicadores financieros los cuales van a ser de gran ayuda para todo el personal.

\section{REFERENCIAS CONSULTADAS}

1. https://creativecomAvalos, F. (2009). Factores determinantes de la competitividad empresarial. Lima: UPC.

2. Bodie, Z., \& Merton, R. (2003). Finanzas. México: Pearson Educación.

3. Cabrera, C., Fuentes, M., \& Cerezo, G. (2017). La gestión financiera aplicada a las organizaciones. 221.

4. Chiavenato, I. (2006). Introducción a la teoría general de la administración. México: McGraw-Hill.

5. Cibrán, P., Prado, C., Crespo, M., \& Huarte, C. (2013). Planificación financiera. Madrid: ESIC.

6. Coulter, M., Robbins, S., \& Decenzo, D. (2013). Fundamentos de Administración. México: Pearson.

7. Cuenca, M. (Marzo de 2014). Universidad Pontificia Comillas ICAI-ICADE. Obtenido de https://repositorio.comillas.edu/xmlui/bitstream/handle/11531/288/TFG000113.pdf ?sequence $=1$

8. De Avila, B., Pérez, H., \& Vega, R. (2016). GESTIÓN FINANCIERA Y COMPETITIVIDAD DE LAS EMPRESAS PRESTADORAS DE SERVICIOS TURÍSTICOS EN LA FRONTERA COLOMBO - VENEZOLANA. Face, 41.

9. Duarte, J., \& Fernández, L. (2011). Finanzas Operativas. México: Panamericana.

10. Escobar, H., Gutiérrez, E., \& Gutiérrez, A. (2007). Hacienda pública un enfoque económico. Medellín: Sello editorial.

11. García, A., Camacho, D., \& Velázquez, N. (2017). Procedimiento de análisis económico-financiero para empresas comercializadoras. Universidad \& ciencia, 244-258. 
12. Guffante, T., Guffante, F., \& Chávez, P. (2016). Investigación Científica: El Proyecto de Investigación. Riobamba: UNACH.

13. Hernández, A., Hernández, A., \& Hernández, A. (2005). Formulación y evaluación de proyectos de inversión. Thomson.

14. Hernández, R., Fernández, C., \& Baptista, M. (2006). Metodología de la investigación. México: McGrawHill.

15. Huerta, J. (1 de 1 de 2014). Universidad de Costa rica. Obtenido de http://academic.uprm.edu/jhuerta/HTMLobj-94/Grupo_Focal.pdf

16. Interior, M. d. (21 de Julio de 2018). La Seguridad Privada, un sector con problemáticas a resolver. Pública FM.

17. Jimenez Cueva, B. M., Erazo Álvarez, J. C., Narváez Zurita, C. I., \& Quevedo Vázquez, J. O. (2019). El balanced scorecard como herramienta de gestión para mejorar la productividad de la industria camaronera: Caso camaronera Biocultivos del Mar (Biomar) Cía. Ltda. Visionario Digital, 88.

18. Kaplan, R., \& Norton, D. (1996). The Balanced Scorecard: Translating Strategy into Action. Boston: Harvard Business School Press.

19. Ledesma, R. (21 de Julio de 2018). 804 empresas de seguridad serán inspeccionadas. El Telégrafo.

20. López, J., \& Sierra, V. (2015). Análisis del impacto del nivel de endeudamiento en la rentabilidad. Obtenido de Universidade Federal de Santa Catarina: http://dvl.ccn.ufsc.br/congresso_internacional/anais/6CCF/33_17.pdf

21. Mathison, L., Gándara, J., Primera, C., \& García, L. (2007). Innovación: Factor clave para lograr ventajas competitivas. Negotium, 46-83.

22. Münch, L. (2010). Administración, gestión organizacional, enfoques y proceso administrativo. México: Pearson.

23. Neill, D., \& Cortez, L. (2018). Procesos y fundamentos de la investigación científica. Machala: UTMACH.

24. Ochoa, C., Sánchez, A., Andocilla, J., Hidalgo, H., \& Medina, D. (2018). El análisis financiero como herramienta clave para una gestión financiera eficiente en las 
medianas empresas comerciales del cantón Milagro. Observatorio de la Economía Latinoamericana.

25. Peña, T., \& Pirela, J. (2007). La complejidad del análisis documental . Información, cultura y sociedad: revista del Instituto de Investigaciones, 55-81.

26. Salazar, A., \& Bajaña, G. (2017). Apalancamiento financiero y su incidencia en la distribución de las utilidades de la empresa Ecuador Overseas Agencies C. A. Observatorio de la economía latinoamericana.

27. Vargas, G., \& Díaz, M. (2017). Crisis y finanzas sustentables: El papel de la deuda y el apalancamiento. Pistas educativas, 271-286.

28.Zamora, J., \& Álvarez, A. (2015). Modelo de Gestión Empresarial Balanced Scorecard. TECTZAPIC. mons.org/licenses/by-nc-sa/4.0/).

\section{REFERENCIAS CONSULTADAS}

1. https://creativecomAvalos, F. (2009). Factores determinantes de la competitividad empresarial. Lima: UPC.

2. Bodie, Z., \& Merton, R. (2003). Finanzas. México: Pearson Educación.

3. Cabrera, C., Fuentes, M., \& Cerezo, G. (2017). La gestión financiera aplicada a las organizaciones. 221.

4. Chiavenato, I. (2006). Introducción a la teoría general de la administración. México: McGraw-Hill.

5. Cibrán, P., Prado, C., Crespo, M., \& Huarte, C. (2013). Planificación financiera. Madrid: ESIC.

6. Coulter, M., Robbins, S., \& Decenzo, D. (2013). Fundamentos de Administración. México: Pearson.

7. Cuenca, M. (Marzo de 2014). Universidad Pontificia Comillas ICAI-ICADE. Obtenido https://repositorio.comillas.edu/xmlui/bitstream/handle/11531/288/TFG000113.pdf ?sequence $=1$ 
8. De Avila, B., Pérez, H., \& Vega, R. (2016). GESTIÓN FINANCIERA Y COMPETITIVIDAD DE LAS EMPRESAS PRESTADORAS DE SERVICIOS TURÍSTICOS EN LA FRONTERA COLOMBO - VENEZOLANA. Face, 41.

9. Duarte, J., \& Fernández, L. (2011). Finanzas Operativas. México: Panamericana.

10. Escobar, H., Gutiérrez, E., \& Gutiérrez, A. (2007). Hacienda pública un enfoque económico. Medellín: Sello editorial.

11.García, A., Camacho, D., \& Velázquez, N. (2017). Procedimiento de análisis económico-financiero para empresas comercializadoras. Universidad \& ciencia, 244-258.

12.Guffante, T., Guffante, F., \& Chávez, P. (2016). Investigación Científica: El Proyecto de Investigación. Riobamba: UNACH.

13. Hernández, A., Hernández, A., \& Hernández, A. (2005). Formulación y evaluación de proyectos de inversión. Thomson.

14. Hernández, R., Fernández, C., \& Baptista, M. (2006). Metodología de la investigación. México: McGrawHill.

15. Huerta, J. (1 de 1 de 2014). Universidad de Costa rica. Obtenido de http://academic.uprm.edu/jhuerta/HTMLobj-94/Grupo_Focal.pdf

16. Interior, M. d. (21 de Julio de 2018). La Seguridad Privada, un sector con problemáticas a resolver. Pública FM.

17. Jimenez Cueva, B. M., Erazo Álvarez, J. C., Narváez Zurita, C. I., \& Quevedo Vázquez, J. O. (2019). El balanced scorecard como herramienta de gestión para mejorar la productividad de la industria camaronera: Caso camaronera Biocultivos del Mar (Biomar) Cía. Ltda. Visionario Digital, 88.

18. Kaplan, R., \& Norton, D. (1996). The Balanced Scorecard: Translating Strategy into Action. Boston: Harvard Business School Press.

19. Ledesma, R. (21 de Julio de 2018). 804 empresas de seguridad serán inspeccionadas. El Telégrafo.

20. López, J., \& Sierra, V. (2015). Análisis del impacto del nivel de endeudamiento en la rentabilidad. Obtenido de Universidade Federal de Santa Catarina: http://dvl.ccn.ufsc.br/congresso_internacional/anais/6CCF/33_17.pdf 
21. Mathison, L., Gándara, J., Primera, C., \& García, L. (2007). Innovación: Factor clave para lograr ventajas competitivas. Negotium, 46-83.

22. Münch, L. (2010). Administración, gestión organizacional, enfoques y proceso administrativo. México: Pearson.

23. Neill, D., \& Cortez, L. (2018). Procesos y fundamentos de la investigación científica. Machala: UTMACH.

24. Ochoa, C., Sánchez, A., Andocilla, J., Hidalgo, H., \& Medina, D. (2018). El análisis financiero como herramienta clave para una gestión financiera eficiente en las medianas empresas comerciales del cantón Milagro. Observatorio de la Economía Latinoamericana.

25. Peña, T., \& Pirela, J. (2007). La complejidad del análisis documental . Información, cultura y sociedad: revista del Instituto de Investigaciones, 55-81.

26. Salazar, A., \& Bajaña, G. (2017). Apalancamiento financiero y su incidencia en la distribución de las utilidades de la empresa Ecuador Overseas Agencies C. A. Observatorio de la economía latinoamericana.

27. Vargas, G., \& Díaz, M. (2017). Crisis y finanzas sustentables: El papel de la deuda y el apalancamiento. Pistas educativas, 271-286.

28.Zamora, J., \& Álvarez, A. (2015). Modelo de Gestión Empresarial Balanced Scorecard. TECTZAPIC. mons.org/licenses/by-nc-sa/4.0/).

(C2019 por los autores. Este artículo es de acceso abierto y distribuido según los términos y condiciones de la licencia Creative Commons Atribución-NoComercial-Compartirlgual 4.0 Internacional (CC BY-NC-SA 4.0) (https://creativecommons.org/licenses/by-nc-sa/4.0/). 